\title{
Inhalt
}

\section{Zweiter Teilband}

\section{Aspekte einer europäischen Sprachgeschichte}

63. Christian Schmitt, Sprach- und Nationenbildung in Westeuropa (bis zur Jahrtausendwende) . . . . . . . . . . . . . . . . .

64. Stefan Sonderegger, Sprachgeschichtliche Aspekte der europäischen Christianisierung . . . . . . . . . . . . . . . . . . 1030

65. Christian Schmitt, Latein und westeuropäische Sprachen . . . . . . . . . 1061

66. Klaus J. Mattheier, Die Herausbildung neuzeitlicher Schriftsprachen . . . 1085

67. Richard Baum, Französisch als dominante Sprache Europas . . . . . . . . . 1107

68. Manfred Görlach, Englisch als neuer Typ von Weltsprache und europäische Nationalsprachen ....................... 1117

69. Baldur Panzer, Gemeinsamkeiten und Unterschiede im Wortschatz europäischer Sprachen . . . . . . . . . . . . . . . . . . . .

70. John Ole Askedal, Gemeinsamkeiten in der grammatischen Struktur europäischer Sprachen ........................ 1136

VIII. Ergebnisse der Sprachgeschichtsforschung zu den historischen Sprachstufen I: Das Althochdeutsche

71. Dieter Geuenich, Soziokulturelle Voraussetzungen, Sprachraum und Diagliederung des Althochdeutschen . . . . . . . . . . . . . . . . . . . 1144

72. Franz Simmler, Phonetik und Phonologie, Graphetik und Graphemik des Althochdeutschen . . . . . . . . . . . . . . . . . . . . 1155

73. Stefan Sonderegger, Morphologie des Althochdeutschen . . . . . . . . . . . 1171

74. Jochen Splett, Lexikologie und Lexikographie des Althochdeutschen . . . 1196

75. Albrecht Greule, Syntax des Althochdeutschen . . . . . . . . . . . . . . . 1207

76. Jochen Splett, Wortbildung des Althochdeutschen . . . . . . . . . . . . . . 1213

77. Alexander Schwarz, Die Textsorten des Althochdeutschen . . . . . . . . . 1222

78. Stefan Sonderegger, Reflexe gesprochener Sprache im Althochdeutschen. . 1231

IX. Ergebnisse der Sprachgeschichtsforschung zu den historischen Sprachstufen II: Das Altniederdeutsche (Altsächsische)

79. Thomas Klein, Soziokulturelle Voraussetzungen und Sprachraum des Altniederdeutschen (Altsächsischen) . . . . . . . . . . . . . . . .

80. Thomas Klein, Phonetik und Phonologie, Graphetik und Graphemik des Altniederdeutschen (Altsächsischen) . . . . . . . . . . . . . . . . . . 1248

81. Heinrich Tiefenbach, Morphologie des Altniederdeutschen (Altsächsischen) 1252 
82. Willy Sanders, Lexikologie und Lexikographie des Altniederdeutschen (Altsächsischen) . . . . . . . . . . . . . . . . . . . . . . . . . . . . . . 1257

83. Irmengard Rauch, Syntax des Altniederdeutschen (Altsächsischen) . . . . .

84. Jürgen Meier/Dieter Möhn, Wortbildung des Altniederdeutschen (Altsächsischen) . . . . . . . . . . . . . . . . . . . . . . . . . . . . . . 1270

85. Willy Sanders, Die Textsorten des Altniederdeutschen (Altsächsischen) . . .

86. Ulrich Scheuermann, Die Diagliederung des Altniederdeutschen (Altsächsischen) . . . . . . . . . . . . . . . . . . . . . . .

87. Willy Sanders, Reflexe gesprochener Sprache im Altniederdeutschen (Altsächsischen) . . . . . . . . . . . . . . . . . . . . . . . .

X. Ergebnisse der Sprachgeschichtsforschung zu den historischen Sprachstufen III: Das Mittelhochdeutsche

88. Ursula Rautenberg, Soziokulturelle Voraussetzungen und Sprachraum des Mittelhochdeutschen . . . . . . . . . . . . . . . . . . . . . .

89. Klaus-Peter Wegera, Grundlagenprobleme einer mittelhochdeutschen Grammatik . . . . . . . . . . . . . . . . . . .

90. Franz Simmler, Phonetik und Phonologie, Graphetik und Graphemik des Mittelhochdeutschen . . . . . . . . . . . . . . . . . . . . . . 1320

91. Siegfried Grosse, Morphologie des Mittelhochdeutschen . . . . . . . . . . .

92. Klaus Grubmüller, Lexikologie und Lexikographie des Mittelhochdeutschen

93. Norbert Richard Wolf, Syntax des Mittelhochdeutschen . . . . . . . . . . .

94. Herta Zutt, Wortbildung des Mittelhochdeutschen . . . . . . . . . . . . .

95. Hannes J. Kästner/Bernd Schirok, Die Textsorten des Mittelhochdeutschen

96. Norbert Richard Wolf, Die Diagliederung des Mittelhochdeutschen . . . .

97. Siegfried Grosse, Reflexe gesprochener Sprache im Mittelhochdeutschen .

98. Ulrike Kiefer, Das Jiddische in Beziehung zum Mittelhochdeutschen . . . .

XI. Ergebnisse der Sprachgeschichtsforschung zu den historischen Sprachstufen IV: Das Mittelniederdeutsche

99. Robert Peters, Soziokulturelle Voraussetzungen und Sprachraum des Mittelniederdeutschen . . . . . . . . . . . . . . . . .

100. Hermann Niebaum, Phonetik und Phonologie, Graphetik und Graphemik des Mittelniederdeutschen . . . . . . . . . . . . . . . . . . 1422

101. John Evert Härd, Morphologie des Mittelniederdeutschen . . . . . . . . .

102. Ingrid Schröder/Dieter Möhn, Lexikologie und Lexikographie des Mittelniederdeutschen . . . . . . . . . . . . . . . . . . . . . . . . 1435

103. John Evert Härd, Syntax des Mittelniederdeutschen . . . . . . . . . . . 1456

104. Gerhard Cordes $(\dagger) /$ Hermann Niebaum, Wortbildung des Mittelniederdeutschen . . . . . . . . . . . . . . . . . . . . . . 1463

105. Jürgen Meier/Dieter Möhn, Die Textsorten des Mittelniederdeutschen . . . 1470

106. Robert Peters, Die Diagliederung des Mittelniederdeutschen . . . . . . . . . 1478

107. Karl Bischoff $(\dagger) /$ Robert Peters, Reflexe gesprochener Sprache im Mittelniederdeutschen . . . . . . . . . . . . . . . . . . . . 
108. Robert Peters, Die Rolle der Hanse und Lübecks in der mittelniederdeutschen Sprachgeschichte . . . . . . . . . . . . . . . . .

109. Timothy Sodmann, Die Verdrängung des Mittelniederdeutschen als Schreib- und Druckersprache Norddeutschlands . . . . . . . . . . . .

XII. Ergebnisse der Sprachgeschichtsforschung zu den historischen Sprachstufen V: Das Frühneuhochdeutsche

110. Hans-Joachim Solms, Soziokulturelle Voraussetzungen und Sprachraum des Frühneuhochdeutschen . . . . . . . . . . . . . . . . . .

111. Norbert Richard Wolf, Phonetik und Phonologie, Graphetik und Graphemik des Frühneuhochdeutschen . . . . . . . . . . . . . . . . .

112. Klaus-Peter Wegera/Hans-Joachim Solms, Morphologie des Frühneuhochdeutschen . . . . . . . . . . . . . . . . . . . . . 1542

113. Dieter Wolf, Lexikologie und Lexikographie des Frühneuhochdeutschen . .

114. Johannes Erben, Syntax des Frühneuhochdeutschen . . . . . . . . . . . . . .

115. Klaus-Peter Wegera/Heinz-Peter Prell, Wortbildung des Frühneuhochdeut-

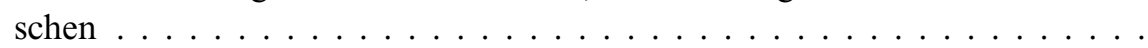

116. Hannes J. Kästner/Eva Schütz/Johannes Schwitalla, Die Textsorten des Frühneuhochdeutschen . . . . . . . . . . . . . . . . . . . 1605

117. Oskar Reichmann, Die Diagliederung des Frühneuhochdeutschen . . . . . .

118. Anne Betten, Zum Verhältnis von geschriebener und gesprochener Sprache im Frühneuhochdeutschen . . . . . . . . . . . . . . . . . . . . . . . . . . . . . . . 1646

119. Rudolf Bentzinger, Die Kanzleisprachen . . . . . . . . . . . . . . . . 1665

120. Joachim Knape, Das Deutsch der Humanisten . . . . . . . . . . . . . . . 1673

121. Frédéric Hartweg, Die Rolle des Buchdrucks für die frühneuhochdeutsche Sprachgeschichte . . . . . . . . . . . . . . . . . . 1682

122. Norbert Richard Wolf, Handschrift und Druck . . . . . . . . . . . . . . 1705

123. Werner Besch, Die Rolle Luthers für die deutsche Sprachgeschichte . . . 1713

XIII. Ergebnisse der Sprachgeschichtsforschung zu den historischen Sprachstufen VI: Das Neuhochdeutsche in seiner Entwicklung vom 17. bis zur Mitte des 20. Jahrhunderts

124. Natalija N. Semenjuk, Soziokulturelle Voraussetzungen des Neuhochdeutschen bis zur Mitte des 20. Jahrhunderts . . . . . . . . . . . . . .

125. Burckhard Garbe, Phonetik und Phonologie, Graphetik und Graphemik des Neuhochdeutschen seit dem 17. Jahrhundert . . . . . . . . . . .

126. Werner Heinrich Veith, Bestrebungen der Orthographiereform im 18., 19. und 20. Jahrhundert . . . . . . . . . . . . . . . . . . . . . . 1782

127. Max Mangold, Entstehung und Problematik der deutschen Hochlautung . 1804

128. Klaus-Peter Wegera, Morphologie des Neuhochdeutschen seit dem 17. Jahrhundert . . . . . . . . . . . . . . . . . . . . . . . . . . 1810

129. Oskar Reichmann, Die Lexik der deutschen Hochsprache . . . . . . . . . . 1818

130. Siegfried Grosse, Die Belebung mittelhochdeutschen Sprachguts im Neu-

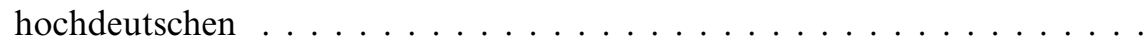


131. Vilmos Ágel, Syntax des Neuhochdeutschen bis zur Mitte des 20. Jahrhun-

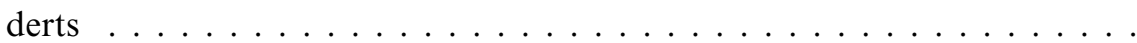

132. Claudine Moulin-Fankhänel, Deutsche Grammatikschreibung vom 16. bis 18. Jahrhundert . . . . . . . . . . . . . . . . . . . . . . . . .

133. Eva-Maria Heinle, Wortbildung des Neuhochdeutschen bis zur Mitte des 20. Jahrhunderts . . . . . . . . . . . . . . . . . . . . . .

134. Heinz Endermann, Die Textsorten des Neuhochdeutschen bis zur Mitte des 20. Jahrhunderts . . . . . . . . . . . . . . . . . . . . . . .

135. Peter Wiesinger, Die Diagliederung des Neuhochdeutschen bis zur Mitte des 20. Jahrhunderts . . . . . . . . . . . . . . . . . . . . . .

136. Klaus J. Mattheier, Die Durchsetzung der deutschen Hochsprache im 19. und beginnenden 20. Jahrhundert: sprachgeographisch, sprachsoziologisch

137. Heinrich Löffler, Gesprochenes und geschriebenes Deutsch bis zur Mitte des 20. Jahrhunderts . . . . . . . . . . . . . . . . . . . . . . . .

138. Utz Maas, Sprache in der Zeit des Nationalsozialismus . . . . . . . . . . 1855

XIV. Entwicklungstendenzen der deutschen Sprache seit der Mitte des 20. Jahrhunderts

139. Lothar Hoffmann, Die Rolle der Fachsprachen seit der Mitte des 20. Jahrhunderts . . . . . . . . . . . . . . . . . . . .

140. Gerd Schank/Johannes Schwitalla, Ansätze neuer Gruppen- und Sondersprachen seit der Mitte des 20. Jahrhunderts . . . . . . . . . . . . . . .

141. Michael Clyne, Varianten des Deutschen in den Staaten mit vorwiegend deutschsprachiger Bevölkerung . . . . . . . . . . . . .

142. Hartmut Schmidt, Entwicklung und Formen des offiziellen Sprachgebrauchs der ehemaligen DDR . . . . . . . . . . . . . . .

143. Heinrich Löffler, Die Rolle der Dialekte seit der Mitte des 20. Jahrhunderts

144. Rainer Wimmer, Sprachkritik in der wissenschaftlichen Diskussion des 20. Jahrhunderts . . . . . . . . . . . . . . . . . . .

145. Rainer Wimmer, Sprachkritik in der Öffentlichkeit seit der Mitte des 20. Jahrhunderts . . . . . . . . . . . . . . . . . . . . . . .

146. Gisela Schoenthal $(\dagger)$, Impulse der feministischen Linguistik für Sprachsystem und Sprachgebrauch . . . . . . . . . . . . . . . . . .

147. Regina Hessky, Entwicklungen der Phraseologie seit der Mitte des 20. Jahr-

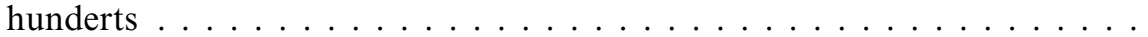

148. Arend Mihm, Die Rolle der Umgangssprachen seit der Mitte des 20. Jahrhunderts . . . . . . . . . . . . . . . . . . . . . .

149. Erich Straßner, Neue Formen des Verhältnisses von Sprache und Visualität seit der Mitte des 20. Jahrhunderts . . . . . . . . . . . . . . . . . .

150. Ruth Römer, Entwicklungstendenzen der Werbesprache seit der Mitte des 20. Jahrhunderts . . . . . . . . . . . . . . . . . . .

151. Norbert Nail, Zeitungssprache und Massenpresse in der jüngeren Geschichte des Deutschen . . . . . . . . . . . . . . . . . . . . . . .

152. Wolfgang Brandt, Sprache in Hörfunk und Fernsehen . . . . . . . . . . .

153. Ulrich Schmitz, Auswirkungen elektronischer Medien und neuer Kommunikationstechniken auf das Sprachverhalten von Individuum und Gesellschaft . . . . . . . . . . . . . . . . . . . . . . . . . 
154. Joachim Born/Wilfried Schütte, Die Stellung des Deutschen in den europäischen Institutionen . . . . . . . . . . . . . . . . . . . . . .

155. Ulrich Ammon, Geltungsverlust und Geltungsgewinn der deutschen Sprache seit der Mitte des 20. Jahrhunderts . . . . . . . . . . . . . . . .

\section{Erster Teilband}

Verzeichnis der Siglen für wissenschaftliche Zeitschriften, Reihen und Sammelwerke

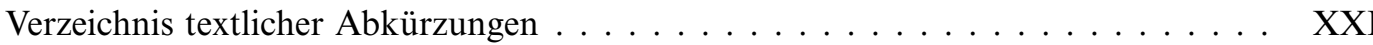
Geleitwort / Foreword / Avant-propos . . . . . . . . . . . . . . XXV Vorwort zur 2., vollständig neu bearbeiteten und erweiterten Auflage . . . . . . . XXIX Vorwort zur ersten Auflage . . . . . . . . . . . . . . . . . . XLI

\section{Deutsche Sprachgeschichte im Rahmen der Kulturgeschichte}

1. Oskar Reichmann, Sprachgeschichte: Idee und Verwirklichung . . . . . . .

2. Peter von Polenz, Deutsche Sprache und Gesellschaft in historischer Sicht . .

3. Joachim Schildt, Deutsche Sprachgeschichte und Geschichte von Institutio-

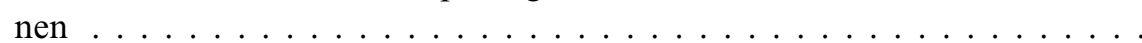

4. Walter Blank, Deutsche Sprachgeschichte und Kirchengeschichte . . . . . .

5. Ruth Schmidt-Wiegand, Deutsche Sprachgeschichte und Rechtsgeschichte bis zum Ende des Mittelalters . . . . . . . . . . . . . . . . . . . . .

6. Ruth Schmidt-Wiegand, Deutsche Sprachgeschichte und Rechtsgeschichte seit dem Ausgang des Mittelalters . . . . . . . . . . . . . . . . .

7. Armin Burkhardt, Deutsche Sprachgeschichte und politische Geschichte. .

8. Jürgen Bolten, Deutsche Sprachgeschichte und Wirtschaftsgeschichte. . . .

9. Klaus-Peter Wegera, Deutsche Sprachgeschichte und Geschichte des All-

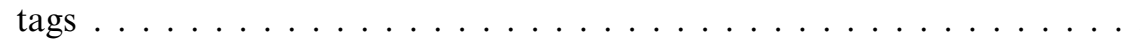

10. Heinrich Cox/Matthias Zender (†), Sprachgeschichte, Kulturraumforschung und Volkskunde. . . . . . . . . . . . . . . . . . . .

11. Karlheinz Jakob, Deutsche Sprachgeschichte und Geschichte der Technik

12. Harald Burger, Deutsche Sprachgeschichte und Geschichte der Philosophie

13. Uwe Pörksen, Deutsche Sprachgeschichte und die Entwicklung der Naturwissenschaften. - Aspekte einer Geschichte der Naturwissenschaftssprache und ihrer Wechselbeziehung zur Gemeinsprache . . . . . . . . . . . . .

14. Werner Koller, Übersetzungen ins Deutsche und ihre Bedeutung für die deutsche Sprachgeschichte . . . . . . . . . . . . . . . . . .

15. Stefan Sonderegger, Geschichte deutschsprachiger Bibelübersetzungen in Grundzügen . . . . . . . . . . . . . . . . . . . . .

16. Hugo Steger, Sprachgeschichte als Geschichte der Textsorten, Kommunikationsbereiche und Semantiktypen . . . . . . . . . . . . . . .

17. Klaus Grubmüller, Sprache und ihre Verschriftlichung in der Geschichte des Deutschen . . . . . . . . . . . . . . . . .

18. Klaus Grubmüller, Gegebenheiten deutschsprachiger Textüberlieferung bis zum Ausgang des Mittelalters . . . . . . . . . . . . . .

19. Wolfgang Schmitz, Gegebenheiten deutschsprachiger Textüberlieferung vom Ausgang des Mittelalters bis zum 17. Jahrhundert . . . . . . . . . . 
II. Sprachgeschichte in gesellschaftlichem Verständnis

20. Andreas Gardt, Die Sprachgesellschaften des 17. und 18. Jahrhunderts . .

21. Ulrike Haß-Zumkehr, Die gesellschaftlichen Interessen an der Sprachgeschichtsforschung im 19. und 20. Jahrhundert . . . . . . . . . . . .

22. Georg Stötzel/Klaus-Hinrich Roth, Das Bild der Sprachgeschichte in deutschen Sprachlehrbüchern . . . . . . . . . . . . . . . . . . .

23. Jochen A. Bär, Die Rolle der Sprachgeschichte in Lexika und sonstigen Werken der Verbreitung kollektiven Wissens . . . . . . . . . . . . . .

24. Klaus-Hinrich Roth, Positionen der Sprachpflege in historischer Sicht . . .

25. Klaus Gloy, Sprachnormierung und Sprachkritik in ihrer gesellschaftlichen Verflechtung . . . . . . . . . . . . . . . . . . . . .

26. Alan Kirkness, Das Phänomen des Purismus in der Geschichte des Deutschen . . . . . . . . . . . . . . . . . . . . . . . .

III. Wissenschaftshistorische Stufen sprachgeschichtlicher Forschung entlang der Zeitlinie

27. Stefan Sonderegger, Ansätze zu einer deutschen Sprachgeschichtsschreibung bis zum Ende des 18. Jahrhunderts . . . . . . . . . . . . . . .

28. Stefan Sonderegger, Sprachgeschichtsforschung in der ersten Hälfte des 19. Jahrhunderts . . . . . . . . . . . . . . . . . . . . .

29. Wolfgang Putschke, Die Arbeiten der Junggrammatiker und ihr Beitrag zur Sprachgeschichtsforschung . . . . . . . . . . . . . . . .

30. Reiner Hildebrandt, Der Beitrag der Sprachgeographie zur Sprachge-

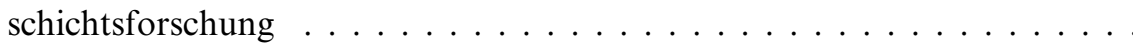

31. Richard Schrodt, Sprachgeschichte in der Sicht strukturalistischer Schulen

32. Willi Mayerthaler, Sprachgeschichte in der Sicht der Generativen Transformationsgrammatik . . . . . . . . . . . . . . . . . .

33. Dieter Cherubim, Sprachgeschichte im Zeichen der linguistischen Pragma-

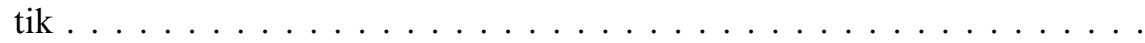

IV. Geschichte und Prinzipien der Sprachgeschichtsforschung nach Beschreibungsebenen

34. Manfred Kohrt, Historische Graphematik und Phonologie . . . . . . . . .

36. Hans-Joachim Solms, Historische Wortbildung . . . . . . . . . . . .

37. Oskar Reichmann/Dieter Wolf, Historische Lexikologie . . . . . . . . . . .

38. Herbert Ernst Wiegand, Historische Lexikographie . . . . . . . . . . . . . .

39. Kurt Gärtner/Peter Kühn, Indices und Konkordanzen zu historischen Texten des Deutschen: Bestandsaufnahme, Typen, Herstellungsprobleme, Be-

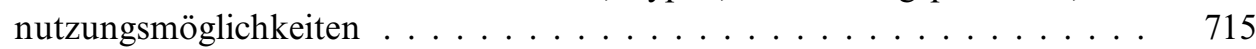

40. Harald Burger/Angelika Linke, Historische Phraseologie . . . . . . . . . 743

41. Franz Hundsnurscher, Historische Syntax . . . . . . . . . . . . . . 755

42. Alfred Bammesberger, Geschichte der etymologischen Forschung seit dem Beginn des 19. Jahrhunderts . . . . . . . . . . . . . . . . . . . . 775

43. Birgit Stolt, Historische Textologie . . . . . . . . . . . . . . . . 786 


\section{Methodologische und theoretische Problemfelder}

44. Thorsten Roelcke, Die Periodisierung der deutschen Sprachgeschichte . . .

45. Ludwig Jäger, Das Verhältnis von Synchronie und Diachronie in der Sprachgeschichtsforschung . . . . . . . . . . . . . . 816

46. Klaus J. Mattheier, Allgemeine Aspekte einer Theorie des Sprachwandels

47. Walter Haas, Ansätze zu einer Theorie des Sprachwandels auf lautlicher Ebene . . . . . . . . . . . . . . . . . . . .

48. Elisabeth Leiss, Ansätze zu einer Theorie des Sprachwandels auf morphologischer und syntaktischer Ebene ..................

49. Gerd Fritz, Ansätze zu einer Theorie des Sprachwandels auf lexikalischer Ebene . . . . . . . . . . . . . . . . . . . . . . .

50. Walter Hoffmann, Probleme der Korpusbildung in der Sprachgeschichtsschreibung und Dokumentation vorhandener Korpora . . . . . . . . . . . .

51. Wolfgang Kleiber, Möglichkeiten historischer Sprachgeographie I: Der hochdeutsche Raum . . . . . . . . . . . . . . . . . . . .

52. Jan Goossens, Möglichkeiten historischer Sprachgeographie II: Der niederdeutsche und niederfränkische Raum . . . . . . . . . . . . . . .

53. Werner Schröder, Editionsprinzipien für deutsche Texte des Früh- und

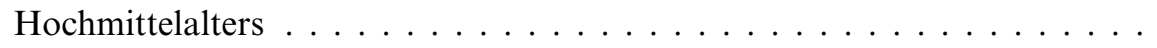

54. Thomas Bein, Editionsprinzipien für deutsche Texte des späten Mittelalters und der frühen Neuzeit . . . . . . . . . . . . . . . . . .

55. Rolf Tarot, Editionsprinzipien für deutsche Texte der Neuzeit I: literarische

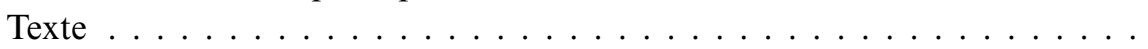

56. Winfried Woesler, Editionsprinzipien für deutsche Texte der Neuzeit II: nichtliterarische Texte . . . . . . . . . . . . . . .

57. Ulrich Krewitt, Probleme des Verstehens altdeutscher Texte und die Möglichkeiten ihrer Übersetzung ins Neuhochdeutsche . . . . . . . . . . . . . . .

VI. Die genealogische und typologische Einordnung des Deutschen

58. Elmar Seebold, Indogermanisch - Germanisch - Deutsch: Genealogische Einordnung und Vorgeschichte des Deutschen . . . . . . . . . . . . . . .

59. Wolfgang Binnig, Der Quellenwert des Gotischen für die sprachgeschichtliche Beschreibung der älteren Sprachstufen des Deutschen . . . . . . . . . .

60. Heinrich Beck, Die germanischen Sprachen der Völkerwanderungszeit . . .

61. Karl-Horst Schmidt, Versuch einer geschichtlichen Sprachtypologie des Deutschen .........................

62. Thorsten Roelcke, Typologische Unterschiede in den Varietäten des Deutschen .......................... 1000

\section{Dritter Teilband (Übersicht über den vorgesehenen Inhalt)}

XV. Ergebnisse der Sprachgeschichtsforschung im Gesamtüberblick I: Pragmatische und soziologische Aspekte

156. Ingo Reiffenstein, Bezeichnungen der deutschen Gesamtsprache

157. Ingo Reiffenstein, Metasprachliche Äußerungen über das Deutsche und seine Subsysteme bis 1800 in historischer Sicht 
158. Werner Wegstein, Die sprachgeographische Gliederung des Deutschen in historischer Sicht

159. Werner Besch, Entstehung und Ausformung der neuhochdeutschen Schriftsprache/Standardsprache

160. Dieter Möhn, Die Stadt in der neueren deutschen Sprachgeschichte I: Hamburg

161. Joachim Schildt, Die Stadt in der neueren deutschen Sprachgeschichte II: Berlin

162. Walter Hoffmann/Klaus J. Mattheier, Die Stadt in der neueren deutschen Sprachgeschichte III: Köln

163. Gaston Van der Elst, Die Stadt in der neueren deutschen Sprachgeschichte IV: Nürnberg

164. Peter Wiesinger: Die Stadt in der neueren deutschen Sprachgeschichte V: Wien

165. Wilfried Seibicke, Fachsprachen in historischer Entwicklung

166. Dieter Möhn, Sondersprachen in historischer Entwicklung

167. Utz Maas, Alphabetisierung. Ein bildungs- und sozialgeschichtlicher Abriß

XVI. Ergebnisse der Sprachgeschichtsforschung im Gesamtüberblick II: Sprachsystematische Aspekte

168. Peter Wiesinger, Lautsystementwicklungen des Deutschen im Bereich von Diphthongierungen, Monophthongierungen, Dehnungen, Kürzungen

169. Heinrich Löffler, Hyperkorrekturen als ein Schlüssel der Sprachgeschichtsforschung

170. Gotthard Lerchner, Konsonantische Lautsystementwicklungen in der Geschichte des Deutschen

171. Dieter Nerius, Graphematische Entwicklungstendenzen in der Geschichte des Deutschen

172. Franz Simmler, Geschichte der Interpunktionssysteme im Deutschen

173. Karin Donhauser, Der Numerus- und Kasusausdruck in der Geschichte des Deutschen

174. Richard Schrodt/Karin Donhauser, Herausbildung und Veränderungen des Tempus- und Modussystems in der Geschichte des Deutschen

175. Johannes Erben, Hauptaspekte der Entwicklung der Wortbildung in der Geschichte des Deutschen

176. Oskar Reichmann, Hauptaspekte des Ausbaus und Umbaus des Wortschatzes in der Geschichte des Deutschen

177. Wolfgang Mieder, Grundzüge einer Geschichte des Sprichtwortes und der Redensart

178. John Evert Härd, Hauptaspekte der syntaktischen Entwicklung in der Geschichte des Deutschen

179. Birgit Stolt, Rhetorikkonzeptionen in der Geschichte der deutschen Sprache

180. Werner Besch, Anredeformen des Deutschen im geschichtlichen Wandel

XVII. Regionalsprachgeschichte

181. Heinz Eickmanns, Aspekte einer niederrheinischen Sprachgeschichte

182. Robert Peters, Aspekte einer Sprachgeschichte des Westfälischen 
183. Robert Peters, Aspekte einer Sprachgeschichte des Sassischen

184. Ulrich Scheuermann, Aspekte einer Sprachgeschichte des Ostfälischen

185. Joachim Gessinger, Aspekte einer Sprachgeschichte des Brandenburgischen

186. Irmtraud Rösler, Aspekte einer Sprachgeschichte des Ostniederdeutschen

187. Klaus J. Mattheier, Aspekte einer rheinischen Sprachgeschichte

188. Hans Ramge, Aspekte einer Sprachgeschichte des Hessischen

189. Gotthard Lerchner, Aspekte einer Sprachgeschichte des Ostmitteldeutschen

190. Helmut Weinacht, Aspekte einer fränkischen Sprachgeschichte

191. Frédéric Hartweg, Die Entwicklung des Verhältnisses von Mundart, deutscher und französischer Standardsprache im Elsaß seit dem 16. Jahrhundert

192. Konrad Kunze, Aspekte einer Sprachgeschichte des Oberrheingebietes bis zum 16. Jahrhundert

193. Stefan Sonderegger, Aspekte einer Sprachgeschichte der deutschen Schweiz

194. Ingo Reiffenstein, Aspekte einer Sprachgeschichte des Bayerisch-Österreichischen bis zum Ausgang des Mittelalters

195. Ingo Reiffenstein, Aspekte einer bayerischen Sprachgeschichte seit der beginnenden Neuzeit

196. Peter Wiesinger, Aspekte einer Sprachgeschichte des Deutschen in Österreich seit der beginnenden Neuzeit

\section{Grundlagen einer literarischen Sprachgeschichte des Deutschen}

197. Anne Betten, Deutsche Sprachgeschichte und Literaturgeschichte

198. Kurt Gärtner, Grundlinien einer literarischen Sprachgeschichte des deutschen Mittelalters

199. Manfred Kaempfert, Grundlinien einer literarischen Sprachgeschichte in neuhochdeutscher Zeit

200. Peter Ernst, Die sprachliche Leistung und Wirkung der deutschen Klassik

201. Thorsten Roelcke, Sprachgeschichtliche Tendenzen des literarischen Experiments im 19. und 20. Jahrhundert

202. Wulf Köpke, Das Sprachproblem der Exilliteratur

203. Anne Betten, Entwicklungen und Formen der deutschen Literatursprache nach 1945

204. Luise Pusch, Ansätze zu einer Geschichte weiblicher Schreibstile

XIX. Das Deutsche im Sprachenkontakt I: Systematische und soziologische Aspekte

205. Els Oksaar, Terminologie und Gegenstand der Sprachkontaktforschung

206. Nikolaus Henkel, Lateinisch/Deutsch

207. Niklas Holzberg, Griechisch/Deutsch

208. N. N., Französisch und Frankoprovenzalisch/Deutsch

209. Max Pfister, Italienisch und Rätoromanisch/Deutsch

210. Karl Mollay (†) / Peter Bassola, Ungarisch/Deutsch

211. Günter Bellmann, Slavisch/Deutsch

212. Ulrike Kiefer, Jiddisch/Deutsch

213. Robert Hinderling, Baltisch/Deutsch

214. Hans-Peter Naumann, Skandinavisch/Deutsch 
215. Gilbert de Smet, Niederländisch/Deutsch

216. Nils Århammar, Friesisch/Deutsch

217. Wolfgang Viereck, Britisches und amerikanisches Englisch/Deutsch

XX. Das Deutsche im Sprachenkontakt II: Aspekte der Sprachgrenzbildung des Deutschen

218. Wolfgang Haubrichs, Geschichte der deutsch-romanischen Sprachgrenze im Westen

219. Stefan Sonderegger, Geschichte der deutsch-romanischen Sprachgrenze im Süden

220. Hermann Scheuringer, Geschichte der deutsch-ungarischen und deutschslavischen Sprachgrenze im Südosten

221. Ernst Eichler, Geschichte der deutsch-slavischen Sprachgrenze im Osten und Nordosten

222. Vibeke Winge, Geschichte der deutsch-skandinavischen Sprachgrenze

223. Ludger Kremer, Geschichte der deutsch-friesischen und deutsch-niederländischen Sprachgrenze

224. Günter Lipold, Geschichte deutscher Sprachinseln in Ost- und Südosteuropa

225. Jürgen Eichhoff, Geschichte deutscher Sprach- und Siedlungsgebiete in Nordamerika

XXI. Deutsche Namengeschichte im Überblick

226. Stefan Sonderegger, Namengeschichte als Bestandteil der deutschen Sprachgeschichte

227. Stefan Sonderegger, Terminologie, Gegenstand und interdisziplinärer Bezug der Namengeschichte

228. Albrecht Greule, Schichten vordeutscher Namen im deutschen Sprachgebiet

229. Friedhelm Debus/Heinz-Günter Schmitz, Überblick über Geschichte und Typen der deutschen Orts- und Landschaftsnamen

230. Wolfgang Kleiber, Die Flurnamen. Voraussetzungen, Methoden und Ergebnisse sprach- und kulturhistorischer Auswertung

231. Albrecht Greule, Überblick über Geschichte und Typen der deutschen Gewässernamen

232. Wilfried Seibicke, Überblick über Geschichte und Typen der deutschen Personennamen

XXII. Register

233. Anja Lobenstein-Reichmann, Sachregister

234. Anja Lobenstein-Reichmann/Silke Bär, Verfasserregister 